\title{
Characteristics of the Covid-19 Pandemic in the United States,
}

\section{0}

\author{
Richard Sauerheber* \\ Palomar Community College, 1140 W Mission Rd., San Marcos, CA 92069, USA
}

Received: 17 September, 2020

Accepted: 24 September, 2020

Published: 25 September, 2020

*Corresponding author: Richard Sauerheber, Palomar Community College, 1140 W Mission Rd., San Marcos, CA 92069, USA, E-mail:RSauerheber@palomar.edu

ORCID: http://orcid.org/0000-0003-4645-2558

https://www.peertechz.com

\begin{abstract}
Time dependent changes in the number of cases of Covid-19, as well as deaths from the disease, are examined and compared to other population parameters including the magnitude of employment across the country. Associations are found that help to explain the tortuous course that the virus infection was able to cause in the U.S.
\end{abstract}

\section{Introduction}

The novel SARS-2 coronavirus spread from China to most of the rest of the world in the year 2020. The virus spread to the U.S. from travelers to China in January, and the earliest significant spread into public communities occurred in March. The virus spread to Europe and from there spread through travelers to New York. It is documented that by this time the virus had mutated to a different form, having a spike protein with an aspartate replaced by a glycine amino acid. This conferred an increased infectivity [1]. In most countries of the world that ordered a National policy of social distancing with mask wearing in public caused the cases to decline and a virtual end to the pandemic, such as first in South Korea [2], and later by countries in Europe after experiencing a huge increase in cases and deaths. California and Washington ordered Statewide lockdowns initially in March. Around Memorial Day these restrictions were lifted with a large return to work allowance. Unlike the biphasic curve that occurred in those countries with widespread ordered restrictions, the U.S. exhibited a resurgence in cases and deaths through July 2020. An analysis of these trends is made here in hopes of assisting the US. with the goal of halting the pandemic.

\section{Methods}

Data on the incidence of new cases of Covid-19, on new deaths from Covid-19, and employment rate are examined as a function of time. Curves were assessed for slope and curvature following principles of the Calculus to see if changing trends could be mathematically described. First derivatives that determine the slopes at any given date, and second derivatives that determine the curvature or cupping of the curves at any particular date were estimated visually. Modified sinusoidal mathematic functions appear to reasonably model the real data.

\section{Results}

Figure 1 shows the daily number of confirmed Covid-19 cases in the U.S. as a function of time from January to July, 2020 [3].

Figure 2 indicates the change in the number of workers employed in the U.S. by companies of various sizes as a function of time over the same time period.

Figure 3 indicates the accumulated death toll in the U.S. from Covid-19 from March to July, 2020.

Figure 4 indicates the number of new deaths per day in the U.S. over the same time frame.

Figure 5 and Figure 6 indicate the number of deaths from Covid-19 in California and in New York State over this time period.

Figure 7 indicates the \% lethality of confirmed cases in Los Angeles and New York City compared to the U.S and the world. 
Figure 8 indicates the percent of cases in the population of Los Angeles, New York City, the U.S., and the world [2].

\section{Discussion}

Analyzing curves with tortuous courses is in the realm of the Calculus. The first derivative of any function represents and computes the slope of the curve at any particular position. The second derivative of a function generates a formula for and represents the curvature or cupping of the curve, which is the slope of the slope for the curve. The second derivative of a curve generating a valley is positive because the downward



Figure 1: The daily number of confirmed Covid-19 cases in the U.S. as a function of time from January to July, 2020 [3]

Employment change by company size (number of workers)

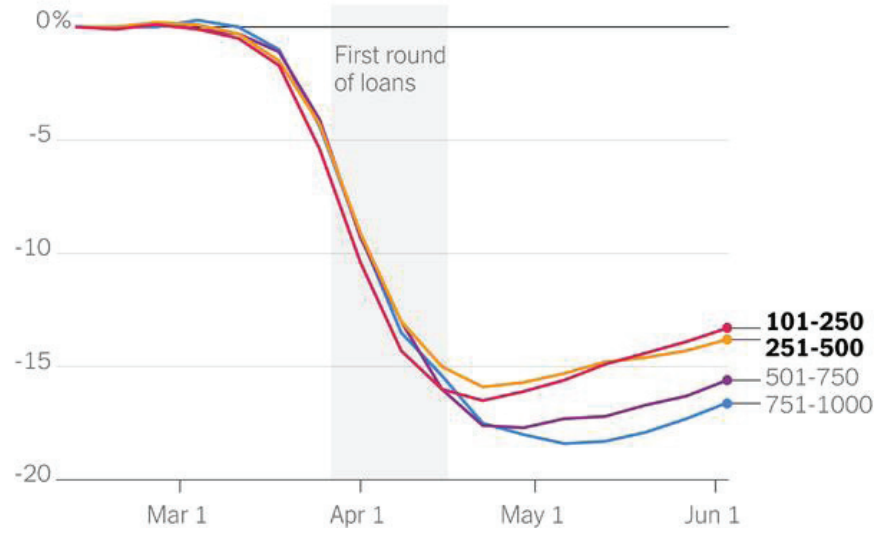

Figure 2: Relative changes in employment in the U.S. as a function of date, as reported in the New York Times, June, 2020

Covid19 Death Toll in U.S. from March 1 to July 18,2020

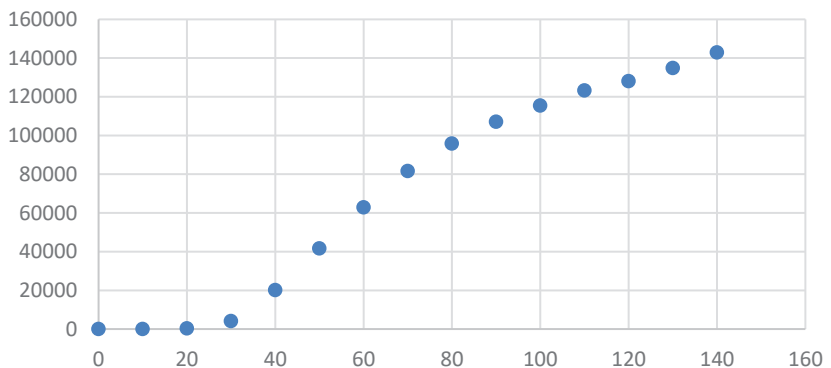

Figure 3: The accumulated death toll in the U.S. due to Covid-19 from March to July, 2020.
Daily New Deaths in the United States Daily Deaths

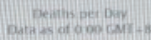

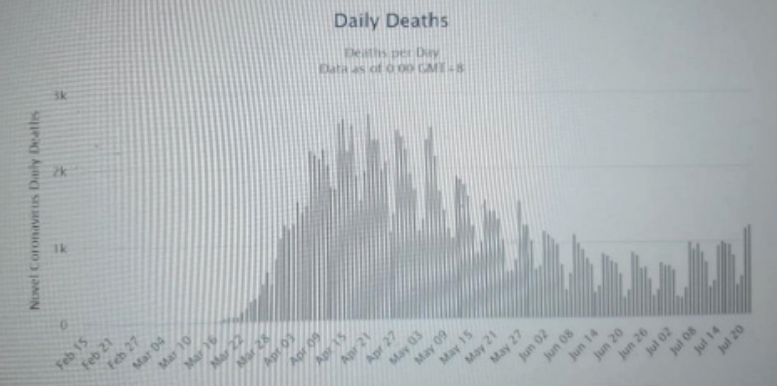

Figure 4: Daily new deaths from Covid-19in the U.S. from March through July,2020.

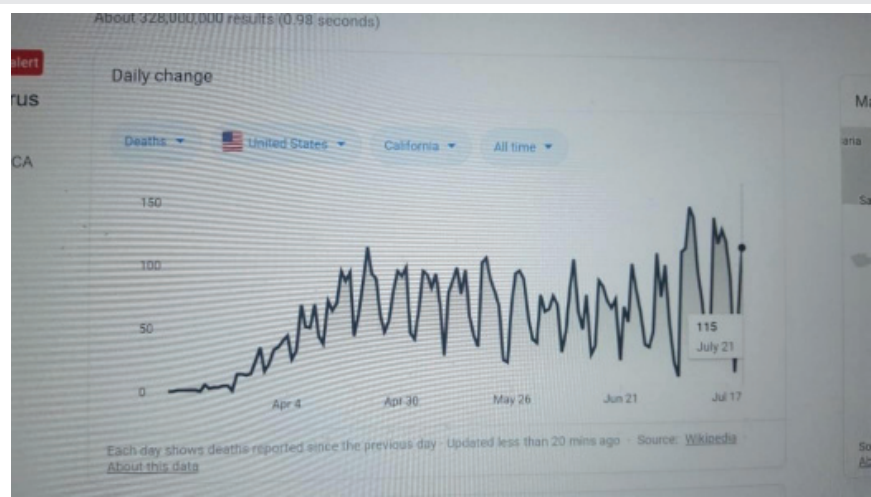

Figure 5: Number of deaths per day in California as a function of time from midMarch to mid-July, 2020. 400,000 confirmed infections accumulated through July 232020 , among a population of 39 million, an incidence or infectivity rate of $1 \%$. With 8,053 total deaths at this date, the virulence or lethality is $1.9 \%$.

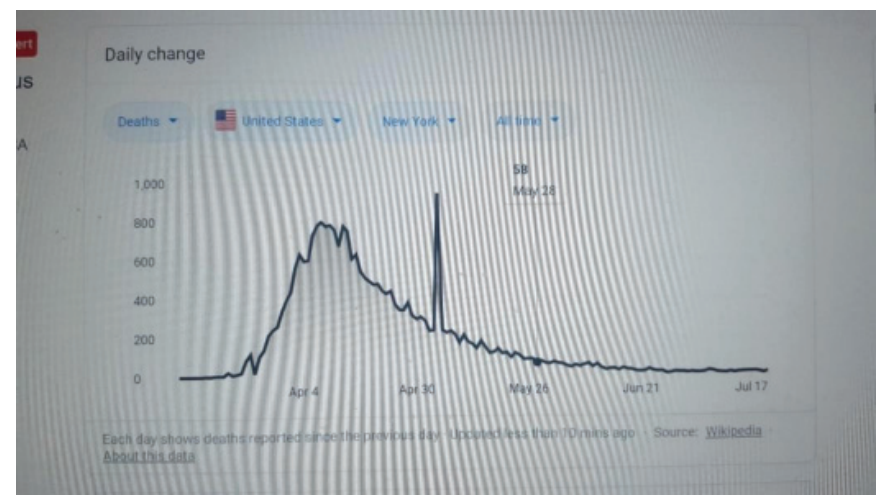

Figure 6: Number of deaths per day in New York State from mid-March to mid-July, 2020. A total of 400,000 infection cases accumulated through July 23 among 19.5 million people, an incidence or infectivity rate of $2.1 \%$. The State total deaths were 25,000 with a virulence or lethality rate of $6 \%$.

slope becomes less negative with time, approaches a slope of zero at the bottom of a well shape, and begins to rise. A curve with a negative second derivative has a negative curvature or cupping such as at the top of a hill where the upward slope begins to decrease in steepness, levels off at the top of the hill shape, and begins a downward negative slope [4].

Notice that in all the curves describing incidence of confirmed Covid-19 cases in this pandemic in the U.S. that in an initial phase of an upward slope in number of cases and deaths is eventually slowed to a point of leveling off on the top 
of a hill shape. This is followed by a downward slope which eventually levels off at the bottom of a valley shape followed by a rise in slope again. This occurred for the U.S. population as a whole, as well as for individual States and cities. For example data for the State of Florida [5] in Figure 9 from March through July, 2020.trace such a curve. This is because the downward cupping with a negative second derivative stops the hill from proceeding higher and brings the curve down while a positive later cupping second derivative causes the downward trend to level off and begin to increase again. The downward cupping followed by upward cupping occurred at specific dates and help to understand the nature of the infectious process in the country.

The initial pandemic was exemplified by a steep rise in number of cases with time in March. This is accompanied with a drop in number of employed people working, as one would expect. This may be due to the virus causing illness with more people staying home from work, coupled with healthy persons staying home to avoid the infection, and any government orders to stay at home. This was followed by a slowing of the rise in cases until a peak was reached and then a drop in daily new cases occurred. This was followed by an increase in employment probably due to recovery from illness, relaxing of stay at home restrictions and requests to open up and to return to work from various officials. This again led to an increase in number of cases perhaps due to infection at work especially in

Covid-19\% Lethality of Confirmed Cases in Los Angeles, New York City, the U.S., and the World, July 23, 2020
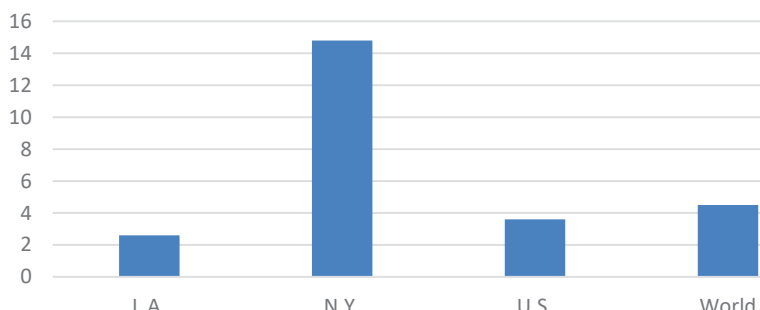

Figure 7: The number of Covid-19 deaths were divided by the number of confirmed cases for the populations of Los Angeles, CA, New York City, NY, as well as for the U.S. and the world population.

Covid-19 \% Incidence in Los Angeles, New York City, the U.S. , and the World, July 23 , 2020



Figure 8: The number of confirmed cases of Covid-19, divided by the corresponding populations of Los Angeles, CA, New York City, NY, the U.S., and the world as of July 23, 2020 (x 100).



work places where closeness or direct contact among workers is necessary. This was followed in time with increased numbers of cases again. Death incidence typically followed the incidence curve by a few weeks since this is the time required for the virus to replicate and spread in an infected victim.

The two strains or mutants of the virus appear to cause differing rates of infection and different lethality. Notice the percent of cases that occurred in Los Angeles and New York are widely different, as are the deaths or lethality rates in the two cities.

The early widespread closure of public schools in the U.S., like elsewhere in the world, has undoubtedly helped mitigate the pandemic. Many reports have indicated that children are infected at a far lower incidence rate than adults and that the lethality or virulence of the virus in children may be lower as well. However it must be emphasized that obviously the spread of the virus through children would be much reduced when social contacts children have while not attending school would be much reduced compared to adults or compared to children in school. As long as a pandemic exists without a cure and a significant lethality ( $2 \%$ in CA and $6-10 \%$ in the NorthEastern U.S.) and a lethality of generally $10 \%$ in the elderly, it is prudent to have off-campus, public schooling consistent with good home schooling practices. In some cases facilities might permit distanced on-campus schooling with students mature enough to consistently be mask-protected.

Los Angeles and New York City appear to have been infected with two different mutants of the novel coronavirus. The East Coast was infected from travelers from Europe with the $G$ virus, while the West Coast was directly infected from travelers from China. The New York City experience thus differed from Los Angeles, where the infectivity of the G form is greeter. NYC in its 5 boroughs or counties had 204,569 cases as of July 23 with 30,187 deaths. This is a death rate or virulence or lethality of $14.8 \%$. Los Angeles at this date had 165,134 confirmed cases with 4,215 deaths, a lethality or virulence of $2.6 \%$..This supports the notion that two forms of the virus infected these locations since the relative proportion of the population that are elderly and more susceptible is not widely different between the two cites, and the capability of the health care systems to treat the condition are also not considered largely different. The difference also however may be somewhat related to the fact that 
the cases appeared more quickly in New York while Los Angeles slowly accumulated cases over this time with rising cases at a time when New York had the infection rate under control. Meanwhile, methods of treating the condition graduated into more frequent use of the RNA replication blocker remdesivir and the cytokine storm blocker dexamethasone that decrease incidence of death in ventilated patients. One possibility is that early control measures ordered in California may have helped prevent the overall incidence from reaching that in New York City. However, it is also possible that the chronic spread of the insidious virus may reach in LA the incidence found in NYC over a long time period. The outcome will shed light on the behavior of the forms of the novel coronavirus in man. NYC population is 8.4 million and LA county is 10 million.

The U.S. lethality or virulence at this date is 144,638 of $4,014,093$ confirmed cases or $3.6 \%$. The world lethality rate as a measure of virulence is 624,890 of $15,274,829$ confirmed cases at $4.5 \%$. The incidence as a measure of infectivity in the U.S. with a population of 328 million is people at $1.2 \%$. The world infectivity or incidence in a population of 7.8 billion is $0.2 \%$. The U.S. lethality as a function of population is $0.044 \%$. The world lethality as a function of population is $0.008 \%$.

The daily Covid-19 positive cases detected in San Diego County, CA are shown in Figure 10. Notice the trend, common in many States and regions, where the upward increase in new cases slows because of the downward cupping of the curve at a time when stay at home orders were honored. This is followed by the expected downward trend in new cases. This is again followed by an upward curvature causing an increase in the appearance of new cases after Memorial Day when restrictions were eased. This is later followed by another downward curvature causing a decrease in the relative number of new cases due to re-imposed restrictions on businesses and public activities again.

These observations are consistent with data for the U.S. and for the world as well, as shown in Figure 11 over the updated more lengthy range from March to September, 2020.

Viral infection rates affect social behavior, and social behavior also directly modifies viral infection rates. In general, when cases are on the rise, it is natural to halt normal employment arrangements, by working at home and to change other social activities. This is not only due to increased sickness but largely also is an attempt to avoid becoming infected. This is followed by a downward trend in number of cases which is then followed by an opening of facilities again. This is followed by an upward trend in new infections again, which again leads to more cautious public activity, and so on. This sinusoidal relation could continue in theory as long as the infection rate is not virtually eliminated before returning to public employment and other activities involving chronic close contacts. Alternatively, the serial repeating curves could be stopped if proper precautions for safety and infection avoidance are undertaken for all public activities in the absence of stay at home orders. The endless cycle reflects lack of social distancing and mask wearing in public after orders for only essential travel are relaxed.

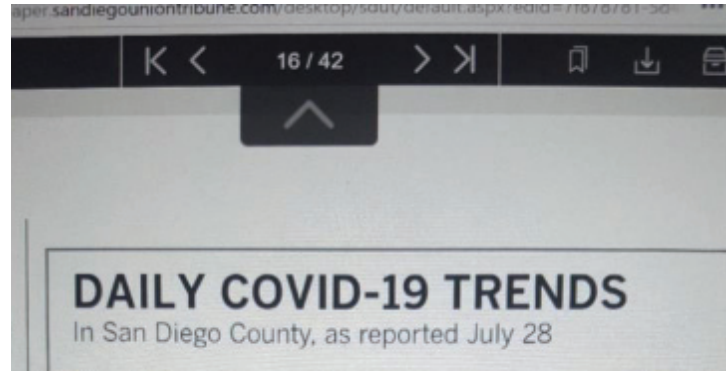

Positive cases by day

\section{Total case}

\section{$\mathbf{2 8 , 2 8 7}$}


. 年

\section{.}

(1)


As Covid cases rise at a rapid rate (time $t=0$ ), employment begins to decline from a maximum. This mitigates the transmission of the virus, and the rate of increase in active cases of infection begins to slow, and at $t=100$ reaches $a$ peak and begins to decline. This decrease in the rate of active infections causes the rate of employment to become less negative so that employment numbers decrease more slowly until employment is at a minimum at $\mathrm{t}=200$. This is followed by continued declines in cases of infection which decrease at a less rapid rate until reaching a minimum at $\mathrm{t}=300$. Employment rises until 360 which causes the steepest rate of increase in infectious cases. This causes the cycle to continue again where increasing cases cause a decrease in the rate of employment again, and so on. The proper way to halt this endless cyclical behavior is to regulate employment so that very safe practices are followed, particularly when employees work in enclosed or relatively close spaces. This would also apply to activities where people congregate closely without proper precautions. Droplets in breath travel substantial distances from merely casual talking. Work involving exertion causes 
droplets to spread much further. Figure 12 shows a theoretic curve of deaths due to the infections described in Figure 13, where an arbitrary $10 \%$ lethality was employed at an average 14 days after infection. The link between public exposure in unregulated workplaces, or unprotected gatherings, with lethal consequences is expected.

Recent data confirms that this sinusoidal cyclical behavior continues repeatedly in the actual pandemic. This is shown for a longer extended time period recently published for San Diego County through September, 2020 (see Figure 14). Two successive sinusoidal waves are clearly seen in these data of confirmed cases averaged in 14 day time intervals.. Notice that the second sine wave is of lower amplitude than the first. This suggests that the public became more engaged in following protective behaviors. A greater percent of the population may be wearing masks and social distancing as employees in the workplace and as citizens in public and social activities.

In a previous study [2] conditions were determined for the chronic inhalation of heated air or steam as a possible mitigant for those infected or exposed to this novel virus. The virus is known to be rendered non-infective after a 20 minute exposure to $\sim 154^{\circ} \mathrm{F}\left(\sim 68^{\circ} \mathrm{C}\right)$, which is possible to achieve with

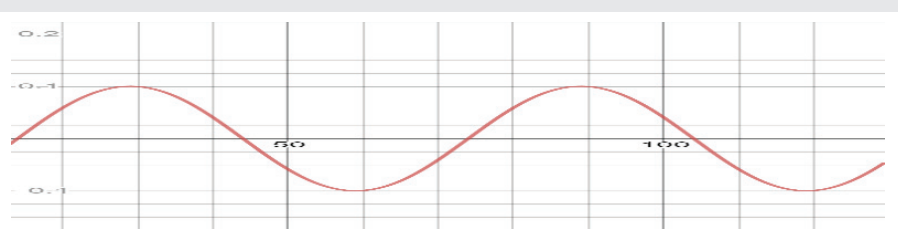

Figure 12: Idealized lethality curve, given by y $3=0.1 \sin (6(t-14))$, assuming $10 \%$ of cases are lethal, and deaths are delayed 14 days after infection. The height of the curve is a $10 \%$ fraction of the infectious cases curve in Figure 11 .

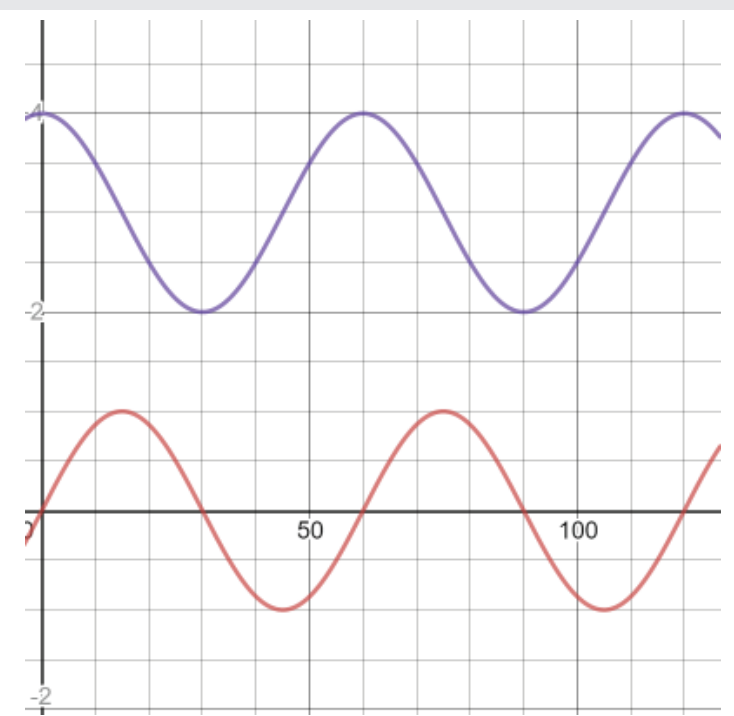

Figure 13: Idealized theoretic relationship between unregulated employment and active cases of Covid-19. Upper curve for number of people gainfully employed is given by $y 1=\cos (6 t)+3$ where 3 could represent 3 million, or 3 thousand, etc. people in a particular population, and $t$ represents time in days. The lower curve for active cases of Covid infection is given by y2 $=\sin (6 t)$. Each hump occurs over a 30 day period of time, approximating the time-dependence of curves seen in the U.S. The height of the cases curve is arbitrary and may refer to a particular unit in a given population.

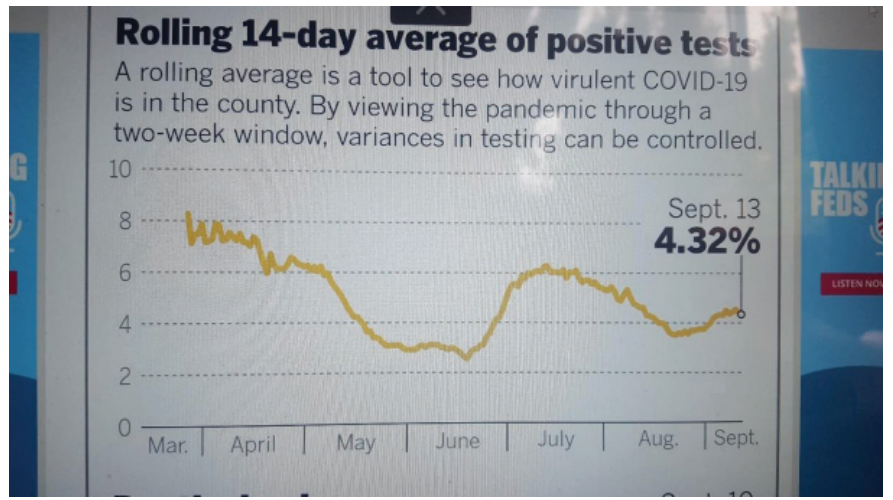

Figure 14: Average positive Covid-19 tests plotted as 14 day averages for San Diego County, CA from March through September 13. As incidence of infection increases, precautions are taken to cause daily incidence to lessen. As incidence lessens, precautions are relaxed and infection incidence increases again. This cycle occurred from March to June and then again from July to September.

a boiled water or heated air without undo discomfort. Although clinical direct studies have not yet been done, some evidence indicates that steam treatment of those with the flu experience significantly decreased convalescent time, and this seems a prudent precaution immediately after one is suspected of being exposed to the novel coronavirus, before initial symptoms appear several days after exposure. Recent studies indicate that remdesivir, the RNA polymerase virus replication inhibitor, and dexamethasone, which impairs inflammation, are clinically effective in shortening recovery times for victims of Covid-19. Finally, recent studies indicate that the flavenoid dietary supplement quercetin (together with vitamin C) is effective at blocking virus insertion into cells and subsequent replication and has been recommended for Covid-19 clinical trials [6].

\section{Recommendations}

These data indicate that when at work or when close to people in public, it is necessary to minimize infection spread by wearing masks and practicing social distancing to prevent significant spread of exhaled breath droplets, wearing of goggles in places where very close contact is unavoidable or in an indoor compact spaces, washing hands often, and bathing and laundering clothes after returning home from work in some cases. The degree to which these measures are followed determines the shape of the incidence (or infectivity) and the death (or lethality) curves for any country. As proof, notice Figure 14 which shows a first wave and then a second wave of infection incidence in San Diego County from March to June corresponding to health warnings which were later followed by relaxation of warnings or a re-opening, and then again from July to September corresponding again to precaution warnings followed by relaxation of warnings. The difference in amplitude of the two waves is undoubtedly due to the fact that a larger segment of the population has become aware of the need to practice precautionary measures. It is hoped that as information is provided across the country, that the U.S. population sinusoidal curves will also become more attenuated than has been found thus far for the successive waves shown in Figures 1 and 11. Attenuating these curves is essential to control the pandemic and to approach our former way of life. 
For example, many countries widely followed mask wearing and distancing protocols which prevented sinusoidal curves of lethal cases. Only 300 total deaths occurred in South Korea for their entire pandemic remaining as a bell-shaped curve.

\section{Acknowledgments}

The people of the State of New York are honored and thanked for the tenacious work in battling and eventually controlling the Covid-19 pandemic there. This confirms that although a virus can dramatically afflict a population, people can greatly control a viral pandemic even in the absence of a vaccine or other cure.

\section{Note added in proof}

As of September, 2020, data from The Johns Hopkins Coronavirus Resource Center reflect a 3.5 fold higher lethality for the Covid-19 pandemic in New York than in California, due presumably to a more virulent virus strain (assuming similar demographics and access to health care). Los Angeles County at this date has a total 6,401 deaths among 262,133 cases, a lethality of $2.4 \%$. Queens County now has a total 6,017 deaths among 71,063 cases at $8.5 \%$ lethality, though fortunately now the pandemic has been effectively controlled in NY. Some public officials argue that the U.S. has turned a corner for the Coronavirus pandemic now, but this is clearly not the case. With total cases in excess of 7 million, and total deaths in excess of 200,000 in the U.S., over 40,000 new confirmed cases and approximately 800 new Covid-19 confirmed deaths continue to occur daily in the country [7]. The U.S. has less than 5 percent of the world's population but more than $20 \%$ of the nearly 1 million reported Covid-19 deaths worldwide (San Diego Union, September 23, 2020, 'U.S. virus death toll tops 200,000'), with a per capita death rate that is $6^{\text {th }}$ in the world, behind Peru, Bolivia, Chile, Spain, and Brazil.

\section{References}

1. Mutated coronavirus shows significant boost in infectivity. 2020 Scripps Research. Link: https://bit.ly/33WtKoA

2. Sauerheber R, McCallum K (2020) Covid-19: Notes on its etiology, pathogenesis, and mediation. Link: https://bit.ly/2RR6GC2

3. Johns Hopkins Coronavirus tracker. Link: https://bit.ly/3mQpwrh

4. Thomas GB, Finney RL (1992) Calculus, $9^{\text {th }}$ edition, Addison Wesley, Reading, Massachusetts. Link: https://bit.ly/33TA2Fr

5. What you need to know now about COVID-19 in Florida. Link: https://bit.ly/3kHEPAA

6. Ruben ML, Biancatelli C, Berrill M, Catravas DJ, Marik PE (2020) Quercetin and Vitamin C: An Experimental, Synergistic Therapy for the Prevention and Treatment of SARS-CoV-2 Related Disease (COVID-19). Front Immunol 11: 1451. Link: https://bit.ly/2Et9Dpa

7. The COVID Tracking Project, The Atlantic, U.S. Daily Cases. Link: https://bit.ly/2FVFz6p

\section{Discover a bigger Impact and Visibility of your article publication with}

\section{Peertechz Publications}

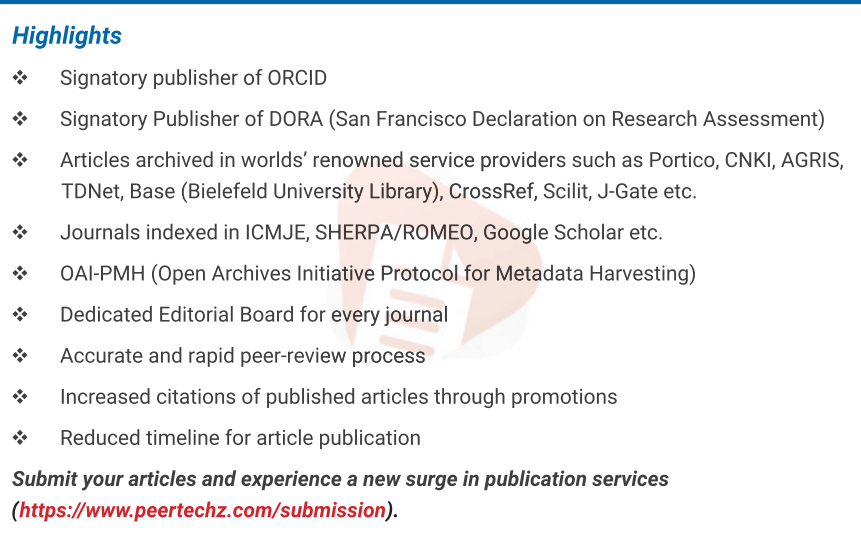

Copyright: @ 2020 Sauerheber R. This is an open-access article distributed under the terms of the Creative Commons Attribution License, which permits unrestricted use, distribution, and reproduction in any medium, provided the original author and source are credited. 